\title{
Fusion and Annihilation of Solitary Waves for a (2+1)-Dimensional Nonlinear System
}

\author{
Ji-Ye Qiang ${ }^{\mathrm{a}, \mathrm{b}, \mathrm{c}}$, Song-Hua Ma ${ }^{\mathrm{b}}$, Qing-Bao Ren ${ }^{\mathrm{b}}$, and Shao-Hua Wang ${ }^{\mathrm{a}}$ \\ a Agronomy College, Nanjing Agricultural University / Key Laboratory of Crop Physiology Ecology \\ in South China, Ministry of Agriculture, Nanjing 210095, China \\ ${ }^{b}$ College of Mathematics and Physics, Lishui University, Lishui, Zhejiang 323000, China \\ c Tobacco College, Yunnan Agricultural University, Kunming 650201, China
}

Reprint requests to S.-H. M.; E-mail: msh6209@yahoo.com.cn

Z. Naturforsch. 65a, 1151 - 1155 (2010); received October 14, 2009 / revised March 21, 2010

\begin{abstract}
In this paper, a new projective equation is used to obtain the variable separation solutions with two arbitrary functions of the (2+1)-dimensional Broek-Kaup system (BKK). Based on the derived solitary wave solutions and by selecting appropriate functions, some novel localized excitations such as fusion and annihilation of solitary waves are investigated.
\end{abstract}

Key words: New Projective Equation; (2+1)-Dimensional BKK System; Fusion; Annihilation.

PACS numbers: 05.45.Yv, 03.65.Ge

\section{Introduction}

Modern soliton theory is widely applied in many natural sciences such as chemistry, biology, mathematics, communication, and in particular in almost all branches of physics like fluid dynamics, plasma physics, field theory, optics, and condensed matter physics [1-7]. Searching for an analytical exact solution to a nonlinear system has long been an important and interesting topic in nonlinear science both for physicists and mathematicians, and various methods for obtaining exact solutions of a nonlinear system have been proposed, for example, the bilinear method, the standard Painlevé truncated expansion, the method of 'coalescence of eigenvalue' or 'wavenumbers', the homogenous balance method, and the mapping method [8-12], etc. The mapping approach is a kind of classic, efficient, and well-developed method to solve nonlinear evolution equations, the remarkable characteristic of which is that we can have many different ansatzs and therefore, a large number of solutions.

In the past, we have solved the exact solutions of some nonlinear systems via the Ricatti equation $\left(\phi^{\prime}=\sigma+\phi^{2}\right)$ mapping method, such as $(1+1)$ dimensional related Schrödinger equation, $(2+1)$-dimensional Generalized Broek-Kaup system, (3+1)-dimensional Burgers system, $(3+1)$-dimensional JimboMiwa system, $(2+1)$-dimensional modified dispersive water-wave system, $(2+1)$-dimensional Boiti-Leon-
Pempinelli system, (2+1)-dimensional Korteweg-de Vries system, (2+1)-dimensional asymmetric NizhnikNovikov-Veselov system, etc. [13-22]. In this paper, with a new projective equation $\left(\phi^{\prime}=\sigma \phi+\phi^{2}\right)$ and a linear variable separation approach, a new family of exact solutions with arbitrary functions of the $(2+1)$ dimensional Broek-Kaup system (BKK) is derived and based on the derived solutions, we find a few novel localized excitations.

The (2+1)-dimensional BKK system is

$$
\begin{aligned}
& u_{t y}-u_{x x y}+2\left(u_{x} u\right)_{y}+2 v_{x x}=0 \\
& v_{t}+2(u v)_{x}+v_{x x}=0 .
\end{aligned}
$$

The BKK system is used to model the nonlinear and dispersive long gravity waves travelling in two horizontal directions in shallow water with uniform depth, and it can also be derived from the celebrated Kadomtsev-Petviashvili (KP) equation by the symmetry constraint [23]. When $y=x$, the $(2+1)$-dimensional BKK system is reduced further to an usual (1+1)dimensional BKK system, which can be used to describe the propagation of long waves in shallow water [24]. Using some suitable dependent and independent variable transformations, Chen and $\mathrm{Li}$ [25] have proved that the $(2+1)$ dimensional BKK system can be transformed to the $(2+1)$-dimensional dispersive long wave equation (DLWE) and (2+1)-dimensional Ablowitz-Kaup-Newell-Segur (AKNS) system. The 
(2+1)-dimensional BKK system has been widely investigated in detail by many researchers [26].

\section{New Exact Solutions of the (2+1)-Dimensional BKK System}

The basic idea of the new equation $\left(\phi^{\prime}=\sigma \phi+\phi^{2}\right)$ mapping approach is as follows. For a given nonlinear partial differential equation (NPDE) with the independent variables $x=\left(x_{0}=t, x_{1}, x_{2}, \cdots, x_{m}\right)$, and the dependent variable $u$, in the form

$$
P\left(u, u_{t}, u_{x_{i}}, u_{x_{i} x_{j}}, \cdots\right)=0,
$$

where $P$ is in general a polynomial function of its arguments, and the subscripts denote the partial derivatives. The solution may be assumed to be in the form

$$
u=\sum_{i=0}^{n}\left\{A_{i}(x) \phi^{i}[q(x)]\right\}
$$

with

$$
\phi^{\prime}=\sigma \phi+\phi^{2}
$$

where $A_{i}(x)$ and $q(x)$ are functions of the indicated argument to be determined, $\sigma$ is an arbitrary constant, and the prime denotes $\phi$ differentiation with respect to $q$. To determine $u$ explicitly, one substitutes (3) and (4) into the given NPDE and collects the coefficients of the polynomials of $\phi$, then eliminates each coefficient to derive a set of partial differential equations for $A_{i}$ and $q$, and solves the system of partial differential equations to obtain $A_{i}$ and $q$. Finally, as (4) is known to possess the solutions

$$
\phi= \begin{cases}-\frac{1}{2} \sigma\left[1+\tanh \left(\frac{1}{2} \sigma q\right)\right], & \sigma \neq 0, \\ -\frac{1}{2} \sigma\left[1+\operatorname{coth}\left(\frac{1}{2} \sigma q\right)\right], & \sigma \neq 0, \\ \frac{-1}{q}, & \sigma=0 .\end{cases}
$$

Substituting $A_{i}, q$, and (5) into (3), one obtains the exact solutions to the given NPDE.

First, let us make a transformation of (1): $v=u_{y}$. Substituting this transformation into (1) yields

$$
u_{y t}+\left[2 u_{x} u_{y}+2 u u_{x y}+u_{x x y}\right]=0 .
$$

Now, we apply the mapping approach to (6). By the balancing procedure, ansatz (3) becomes

$$
u(x, y, t)=f(x, y, t)+g(x, y, t) \phi(q),
$$

where $f, g$, and $q$ are functions of $(x, y, t)$ to be determined. Substituting (7) and (4) into (6) and collecting coefficients of the polynomials in $\phi$, then setting each coefficient to zero, we have

$$
f=-\frac{1}{2} \frac{q_{t}+q_{x x}+q_{x}^{2} \sigma}{q_{x}}, \quad g=-q_{x}
$$

with

$$
q=\chi(x, t)+\varphi(y),
$$

where $\chi(x, t)$ and $\varphi(y)$ are two arbitrary functions of the indicated arguments.

Case 1. For $\sigma \neq 0$, we can derive the following solitary wave solutions of (1):

$$
\begin{aligned}
& u_{1}=-\frac{1}{2} \frac{\chi_{t}+\chi_{x x}-\chi_{x}^{2} \sigma \tanh \left[\frac{1}{2} \sigma(\chi+\varphi)\right]}{\chi_{x}}, \\
& v_{1}=\frac{1}{4} \chi_{x} \varphi_{y} \sigma^{2}\left\{1-\tanh \left[\frac{1}{2} \sigma(\chi+\varphi)\right]^{2}\right\}, \\
& u_{2}=-\frac{1}{2} \frac{\chi_{t}+\chi_{x x}-\chi_{x}^{2} \sigma \operatorname{coth}\left[\frac{1}{2} \sigma(\chi+\varphi)\right]}{\chi_{x}}, \\
& v_{2}=\frac{1}{4} \chi_{x} \varphi_{y} \sigma^{2}\left\{1-\operatorname{coth}\left[\frac{1}{2} \sigma(\chi+\varphi)\right]^{2}\right\}
\end{aligned}
$$

with two arbitrary functions being $\chi(x, t)$ and $\varphi(y)$.

Case 2. For $\sigma=0$, we can derive the following variable separated solution of (1):

$$
\begin{aligned}
& u_{5}=-\frac{1}{2} \frac{\chi_{t}+\chi_{x x}}{\chi_{x}}+\frac{\chi_{x}}{\chi+\varphi}, \\
& v_{5}=-\frac{\chi_{x} \varphi_{y}}{(\chi+\varphi)^{2}}
\end{aligned}
$$

with two arbitrary functions being $\chi(x, t)$ and $\varphi(y)$.

\section{Some Localized Excitations in \\ (2+1)-Dimensional BKK System}

In this section, we mainly discuss some localized coherent excitations in the (2+1)-dimensional BKK system. For simplification, we only discuss the field $v_{2}$ of (13), namely

$$
V=v_{2}=\frac{1}{4} \chi_{x} \varphi_{y} \sigma^{2}\left\{1-\operatorname{coth}\left[\frac{1}{2} \sigma(\chi+\varphi)\right]^{2}\right\} \text {. }
$$


(a)

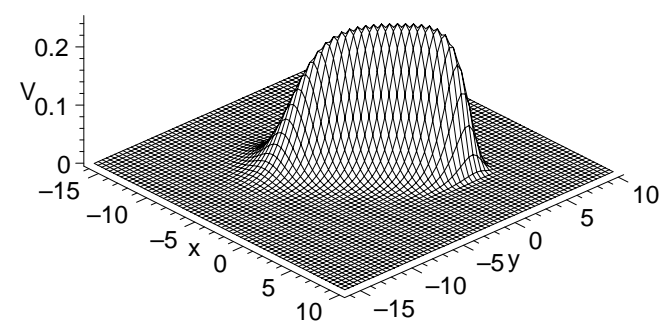

(b)

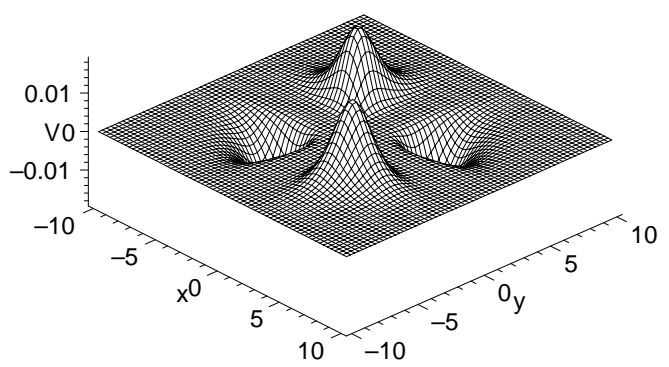

Fig. 1. (a) Plot of a dromion structure for the physical quantity $V$ given by the solution (16) with the choice (17) and $k=1$, $t=0$. (b) Plot of a multi-dromion structure for the physical quantity $V$ given by the solution (16) with the choice (18) and $k=1, t=0$.

(a)

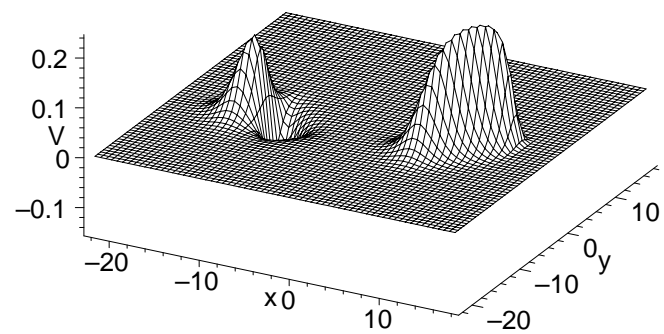

(c)

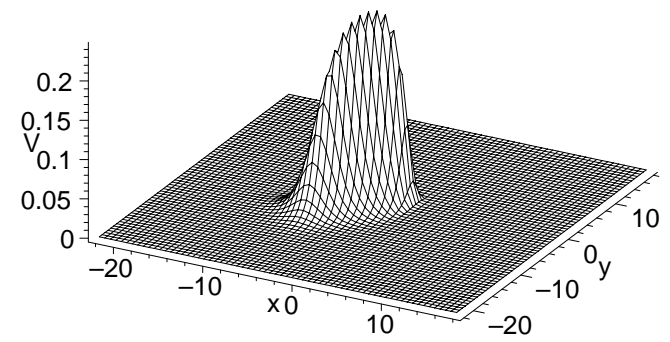

(b)

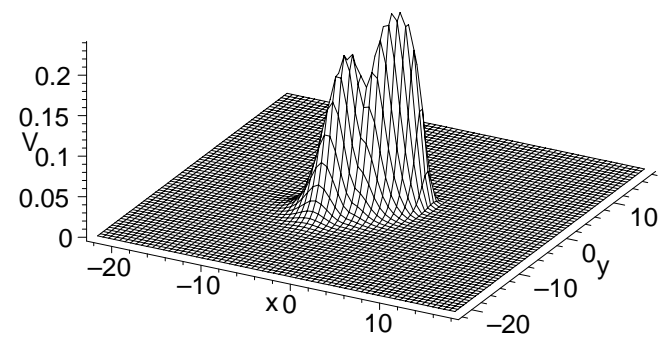

(d)

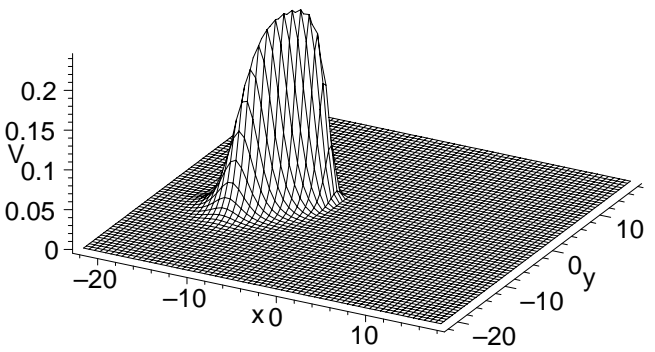

Fig. 2. Evolutional profile of a two single-soliton fusion into one soliton for the solution $V$ expressed by (16) with condition (17) at different times (a) $t=-5$, (b) $t=-2$, (c) $t=2$, (d) $t=5$, respectively.

\subsection{Dromion Excitations}

In $(2+1)$ dimensions, one of the most important nonlinear solutions is the dromion excitation, which is localized in all directions exponentially. For instance, if we choose $\chi$ and $\varphi$ as

$$
\begin{aligned}
& \chi=0.0001+0.1 \exp (x+k t), \\
& \varphi=0.0001+0.1 \exp (y),
\end{aligned}
$$

we can obtain a dromion structure for the physical quantity $V$ of (16) presented in Figure 1a with fixed parameters $k=1$ and $t=0$. If we choose $\chi$ and $\varphi$ as

$$
\chi=1+10 \sec (x+k t), \quad \varphi=1+10 \operatorname{sech}(y),
$$

we can obtain a multi-dromion structure for the physical quantity $V$ of (16) presented in Figure 1b with fixed parameters $k=1$ and $t=0$.

\subsection{The Fusion and Annihilation of Solitons}

Now, we focus our attention on these intriguing fusion and annihilation phenomena for the solitary wave solutions $V$, which may exist under certain circumstances. For instance, when we select the arbitrary functions $\chi$ and $\varphi$ to be

$$
\begin{aligned}
& \chi=0.001+\exp (x+t)+0.01 \operatorname{sech}(x-t), \\
& \varphi=0.001+\tanh (y),
\end{aligned}
$$

and substitute (19) for (16), we can obtain a new kind of solitary solution of (1). Figure 2 shows an evolutional profile corresponding to the physical quantity $V$ of the two-dromion solution expressed by (16), exhibiting a fusion phenomenon for the two solitons. From Figure 2, we can clearly see that the two single-solitons fuse to one soliton finally. 
(a)

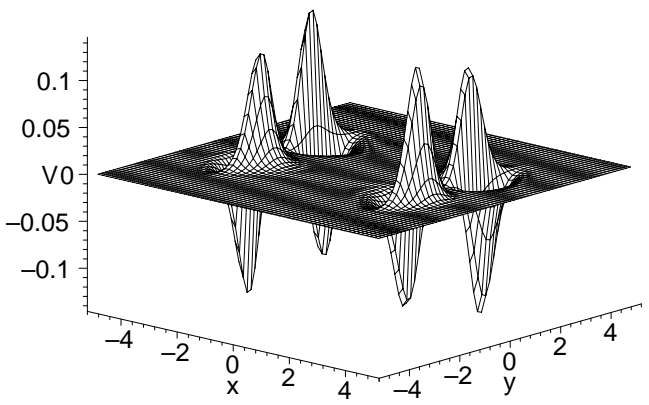

(c)

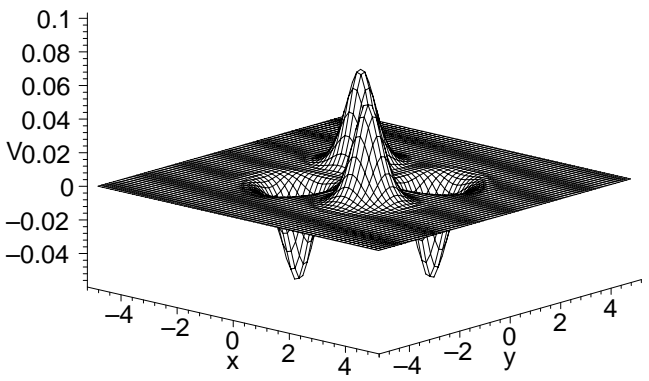

(e)

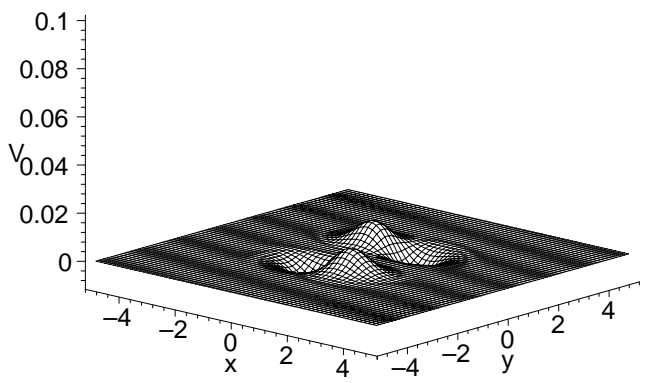

(b)

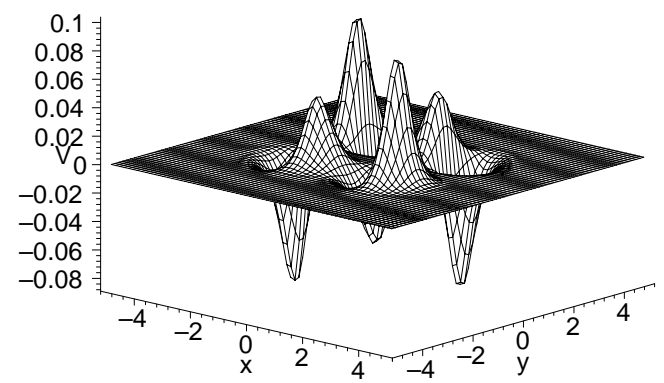

(d)

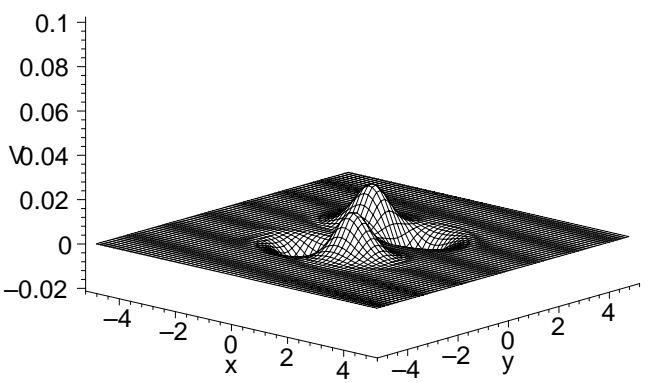

(f)

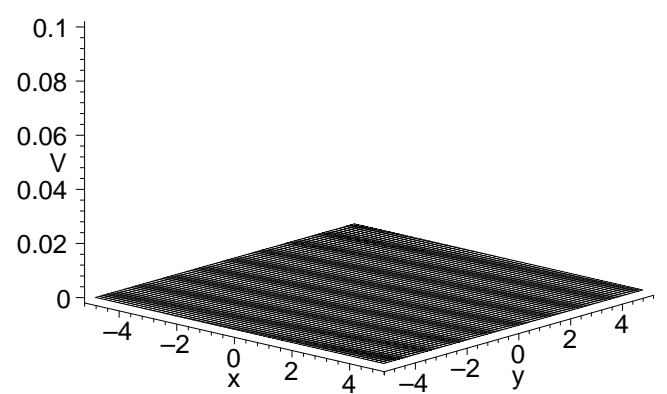

Fig. 3. Plot of the annihilation of solitons for the solution $V$ by (16) under the condition (20) at different times (a) $t=-7$, (b) $t=0$, (c) $t=2$, (d) $t=3$, (e) $t=5$, (f) $t=7$, respectively.

Just like other particles, solitons can also be annihilated in some appropriate conditions. For example, when choosing $\chi(x, t)$ and $\varphi(y)$ in solution (16) to be

$$
\chi=1+\operatorname{sech}\left(x^{2}+t\right), \quad \varphi=1+\operatorname{sech}\left(y^{2}\right),
$$

we can see that the annihilation of solitons for the physical quantity $V$ of (16) under the condition (20) presented in Figure 3 with fixed parameters $t=-7,0$, 2, 3, 5, 7. From Figure 3, we find that the amplitude and shape of the solitons become smaller and smaller after interactions, finally, they reduce to zero.

\section{Summary and Discussion}

In the past, many authors have solved the exact solutions of some nonlinear systems via the Ricatti equation $\left(\phi^{\prime}=\sigma+\phi^{2}\right)$ mapping method. In summary, via a new projective equation $\left(\phi^{\prime}=\sigma \phi+\phi^{2}\right)$ and a linear variable separation approach, we find some new exact solutions of the $(2+1)$-dimensional Broek-Kaup system. Based on the derived solitary wave excitation, we have studied the fusion and annihilation phenomena of solitons. And we can see that solitons have the same characteristics as other particles in many aspects.

Although we have given out some soliton fusion and annihilation phenomena in the (2+1)-dimensional case, it is obvious that there are still many significant and interesting problems to be further discussed. As the authors [27] have pointed out in the $(1+1)$-dimensional cases: What is the necessary and sufficient condition for soliton fusion and fission? What is for soliton elastic and nonelastic interactions? What is the general equation for the distribution of the energy and momentum after soliton fusion and soliton fission? How can 
we use the soliton fusion and soliton fission of integrable models to investigate the observed soliton fusion and soliton fission in the experiments? These are all the pending issues to be further studied.

\section{Acknowledgements}

The authors would like to thank Professor Jie-Fang Zhang for his fruitful and helpful suggestions. This

[1] S. Y. Lou, Phys. Rev. Lett. 80, 5027 (1998).

[2] D. J. Zhang, Chaos, Solitons, and Fractals 22, 1333 (2005).

[3] R. Camassa and D. D. Holm, Phys. Rev. Lett. 71, 1661 (1993).

[4] J.P. Fang and C. L. Zeng, Z. Naturforsch. 60a, 245 (2005).

[5] S. L. Zhang and J. N. Li, Chin. Phys. Lett. 24, 1433 (2007).

[6] P.Z. Wang, P.Z. Wang, and C.Z. Qu, Chin. Phys. Lett. 22, 1029 (2005).

[7] D. J. Zhang, Chin. Phys. Lett. 23, 2349 (2006).

[8] J. Hietarinta, Phys. Lett. A 149, 113 (1990).

[9] S. Y. Lou, J. Lin, and X. Y. Tang, Eur. Phys. J. B 22, 473 (2001).

[10] D. W. C. Lai and K. W. Chow, J. Phys. Soc. Jpn. 70, 666 (2001).

[11] J.F. Zhang, Commun. Theor. Phys. (Beijing, China) 37, 277 (2002).

[12] J. P. Fang and C. L. Zheng, Z. Naturforsch. 60a, 245 (2005).

[13] J. P. Fang and C. L. Zheng, Acta Phys. Sin. 54, 2991 (2005).

[14] S. H. Ma, J. P. Fang, and C. L. Zheng, Chaos, Solitons, and Fractals 40, 210 (2009). work has been supported by the Natural Science Foundation of Zhejiang Province (Grant Nos. Y610025, Y6090545), the Scientific Research Fund of Zhejiang Provincial Education Department of China (Grant No. 20070568), the undergraduate innovation project of science and technology of Zhejiang Province, the project of seeding grants of Zhejiang Province, and Natural Sciencey Foundation of Zhejiang Lishui University (Grant No. KZ09005).

[15] S.H. Ma, X.H. Wu, J.P. Fang, and C.L. Zheng, Z. Naturforsch. A 61, 249 (2006).

[16] S. H. Ma and J. P. Fang, Acta Phys. Sin. 55, 37 (2006).

[17] S. H. Ma, J. P. Fang, and C. L. Zheng, Z. Naturforsch. 62a, 8 (2007).

[18] S. H. Ma, J. Y. Qiang, and J.P. Fang, Acta Phys. Sin. 56, 0620 (2007).

[19] S. H. Ma, J. P. Fang, and C. L. Zheng, Acta Phys. Sin. 56, 4319 (2007).

[20] S. H. Ma, J. Y. Qiang, and J. P. Fang, Commun. Theor. Phys. (Beijing, China) 48, 662 (2007).

[21] S. H. Ma, J.P. Fang, and C. L. Zheng, Z. Naturforsch. 63a, 121 (2008).

[22] J. B. Li, C.L. Zheng, and S.H. Ma, Z. Naturforsch. 63a, 323 (2008).

[23] S. Y. Lou and X. B. Hu, J. Math. Phys. 38, 6401 (1997).

[24] V.E. Zakharov and L. Li, Appl. Mech. Tech. Phys. 9, 190 (1998).

[25] C. L. Chen and Y. S. Li, Theor. Phys. 38, 129 (2002).

[26] J.P. Ying and S. Y. Lou, Z. Naturforsch. 56a, 619 (2001).

[27] S. Wang, Y. S. Xu, and S. Y. Lou, Chin. Phys. 14, 1049 (2003). 